\title{
DISPNÉIA EM INDIVÍDUOS COM ASMA, RINITE ALÉRGICA E RESPIRAÇÃO ORAL
}

\section{Dyspnea in patients with asthma, allergic rhinitis and mouth breathing}

\author{
Silvia Márcia Andrade Campanha ${ }^{(1)}$, Maria Jussara Freire Fontes (2), \\ Júlia Larissa Ferreira dos Santos ${ }^{(3)}$
}

\begin{abstract}
RESUMO
Objetivo: avaliar a presença de dispnéia durante atividades físicas, de fala e sua associação. Método: a população alvo foi composta de 24 indivíduos com diagnóstico médico prévio de asma, rinite alérgica e respiração oral, onde aplicou-se oralmente em cada individuo o Questionário de Dispnéia traduzido por Cukier (1998), composto por 30 questões que permitiu a quantificação da dispnéia em atividades de fala, físicas e associadas. Resultados: pela análise do questionário verificou-se maior freqüência da dispnéia durante a realização das atividades físicas associadas à fala, seguidas das atividades físicas isoladas e depois associadas as atividades de fala. Conclusões: as maiores queixas respiratórias foram encontradas nas atividades físicas associadas à fala, seguidas das atividades físicas isoladas. Já nas atividades comunicativas, apenas as atividades que exigiam maior intensidade vocal foram relatadas como dificultosas por alguns indivíduos.
\end{abstract}

DESCRITORES: Respiração Bucal; Asma; Dispnéia

\section{INTRODUÇÃO}

A asma se caracteriza por uma inflamação crônica das vias aéreas e obstrução ao fluxo aéreo, sendo considerada uma doença crônica e geralmente reversível que gera sofrimento a pacientes e familiares e evolui eventualmente para o óbito ${ }^{1}$

Já a rinite alérgica ocorre perante exposição à alérgenos que causam inflamação da mucosa nasal, e pode estar presente de forma persistente ou intermitente e variar de intensidade ${ }^{2}$. Sua

(1) Fonoaudióloga; Professora da Graduação de Fonoaudiologia da Faculdade de Estudos Administrativos - Centro de Gestão Empreendedora; Mestre em Ciências da Saúde do Instituto de Previdência dos Servidores do Estado de Minas Gerais.

(2) Pediatra; Professora Adjunta de Pediatria e Pneumologia Pediátrica da Faculdade de Medicina da Universidade Federal de Minas Gerais; Professora da Pós-Graduação em Ciências da Saúde da Criança e do Adolescente da Faculdade de Medicina da Universidade Federal de Minas Gerais

(3) Fonoaudióloga; Fonoaudióloga da APAE do município de Três Marias e da Prefeitura Municipal de Biquinhas, Minas Gerais; Especialização em Audiologia pela Faculdade de Estudos Administrativos - FEAD-MG.

Conflito de interesses: inexistente prevalência está em torno de 10,25 \% da população em geral. Sendo considerada como uma das etiologias obstrutivas mais freqüentes na população infantil ${ }^{2-4}$.

A ligação da asma com a rinite alérgica pode ser identificada por vários aspectos, sendo eles: epidemiológico, histológico, fisiológico e imunológico, sendo sua coexistência freqüente na população ${ }^{5}$.

O processo inflamatório da mucosa das vias aéreas, decorrente tanto da asma quanto da rinite, ocasiona a obstrução nasal e conseqüente respiração oral, levando ao surgimento concomitante da asma, rinite e respiração oral $(\mathrm{RO})^{6-8}$.

O respirador oral é um indivíduo que, possuindo um padrão de respiração nasal insuficiente, respira pela boca, na maior parte do tempo ${ }^{9}$. Sua etiologia pode ser classificada em obstrutiva ou não-obstrutiva que e causada por hábito, já as causas obstrutivas são: rinites, hipertrofias de cornetos, trauma nasal, desvio de septo, malformações nasais, polipose nasal, tumores da cavidade nasal e rinofaringe, hipertrofia de amígdalas e tonsila faríngea ${ }^{10}$.

Devido às diversas alterações que a RO causa ao indivíduo, é considerada atualmente como uma síndrome. As alterações mais comumente descritas na literatura são: alterações orofaciais, posturais, 
oclusais e distúrbios de comportamento9. Verificase também alteração na capacidade pulmonar, diminuição do pico de fluxo expiratório e da expansibilidade torácica, além da dificuldade inspiratória usual ${ }^{11}$. Devido a tais alterações pulmonares a queixa de dispnéia ou "falta de ar" torna-se freqüente em indivíduos respiradores orais.

Alguns autores relatam a repercussão destas alterações na qualidade de vida dos indivíduos, afetando diretamente seu bem estar físico, psicológico, relacionamento social e desempenho escolar 7,9,12-14. E, visando minimizar essas conseqüências, o tratamento do respirador oral deve constar de uma equipe interdisciplinar ${ }^{15}$.

Diante da associação entre RO e suas alterações pulmonares que levam a dispnéia, o presente estudo tem como objetivo descrever a dispnéia em situações de atividade físicas, de fala e de sua associação, visando assim, um maior conhecimento das alterações decorrentes da RO e conseqüente melhor qualidade de vida.

\section{MÉTODO}

Trata-se de um estudo descritivo realizado no Ambulatório de Pneumologia Pediátrica do Hospital das Clínicas da Universidade Federal de Minas Gerais e no consultório particular da pesquisadora, cuja população alvo foi a de 169 crianças e adolescentes asmáticos, de 6 a 15 anos de idade, com teste cutâneo positivo a pelo menos um dos alérgenos pesquisados, com quadro clínico de rinite alérgica e respiração oral. Esses pacientes foram atendidos no Pronto Atendimento Municipal Padre Eustáquio, onde receberam os diagnósticos acima descritos.

Foram excluídos pacientes com hipertrofia da tonsila faríngea, com desvio de septo, pólipos nasais, infecções das vias aéreas superiores e inferiores, que podiam interferir na avaliação da asma, da rinite alérgica e da respiração oral. Foram excluídos, também, os pacientes com doenças subjacentes de qualquer natureza, bem como os com volume expiratório forçado no primeiro segundo (VEF1) ou com a relação VEF1/CVF (CVF= capacidade vital forçada) menor que $40 \%$ do valor previsto que caracteriza asma severa, fator de exclusão do estudo.

Após a aplicação dos critérios de inclusão e exclusão foram selecionados 24 pacientes portadores de asma, rinite alérgica e respiração oral que foram submetidos ao Questionário Especifico Para Dispnéia traduzido por Cukier ${ }^{16}$. Trata-se de um questionário composto por 30 perguntas que se dividem em três grupos sendo que as perguntas de 01 a 10 referem-se à presença de dispnéia relacionada a esforço físico (andar, vestir, comer, tomar banho, caminhar, subir escadas, fazer esportes, correr e levantar peso), de 11 a 20 dispnéia relacionada a atividades de fala (conversar, cantar, aumentar o tom de voz, falar em público, com TV ligada, no carro, num restaurante, em lugar ruidoso e com autoridade) e de 21 a 30 na associação dessas duas atividades (falar andando, vestindo, subindo ladeira, degraus, realizando esportes, tomando banho, levantando peso e correndo).

As respostas seguem em uma escala de pontos de acordo com a dificuldade apresentada pelo paciente, onde 1 refere a não apresentar dispnéia durante a realização da atividade, 3 se sente ocasionalmente dificuldade de respirar e 5 se sente sempre, caso a atividade não seja realizada pelo entrevistado corresponde ao número 9.

Este estudo foi aprovado pelo Comitê de Ética em Pesquisa da Secretaria Municipal de Saúde de Belo Horizonte (protocolo 060/2005) e Universidade Federal de Minas Gerais (protocolo no ETIC 0169/06). Os responsáveis pelos participantes assinaram o Termo de Consentimento Livre e Esclarecido.

Foi utilizado o programa Epi-Info para análise descritiva dos dados, considerando-se os valores percentuais e numéricos para as variáveis pesquisadas.

\section{RESULTADOS}

A amostra foi constituída por 24 indivíduos de ambos os gêneros, sendo $8 \%$ (4) do gênero feminino e $92 \%$ (20) do masculino, com idades entre 5 a 15 anos.

Na Tabela 1, observam-se os resultados obtidos pelos participantes em relação à dispnéia durante a realização de atividades físicas, onde maior parte dos participantes $(75 \%)$ relataram maior comprometimento respiratório nas atividades de correr e realizar esportes.

$\mathrm{Na}$ Tabela 2, verifica-se menor relato de dispnéia nas atividades de fala, sendo o aumento da voz a atividade de fala mais freqüentemente associada à dispnéia.

$\mathrm{Na}$ Tabela 3 encontram-se descritos os resultados envolvendo as atividades físicas associadas às atividades de fala. A maior presença de dispnéia foi encontrada nestas atividades destacando-se falar subindo ladeira, degraus, carregando peso e correndo. 
Tabela 1 - Descrição dos resultados obtidos em relação à dispnéia durante a realização de atividades físicas

\begin{tabular}{lcccccc}
\hline \multirow{2}{*}{ Atividades Físicas } & \multicolumn{7}{c}{ Presença de Dispnéia } \\
\cline { 2 - 7 } & \multicolumn{2}{c}{ Ausência } & \multicolumn{2}{c}{ Ocasionalmente } & \multicolumn{2}{c}{ Sempre presente } \\
\cline { 2 - 7 } & $\%$ & $\mathbf{N}$ & $\%$ & $\mathbf{N}$ & $\%$ & $\mathbf{N}$ \\
\hline 1- Andar & 79 & 19 & 21 & 19 & 0 & 0 \\
2- Vestir & 79 & 19 & 12,5 & 3 & 8,5 & 2 \\
3- Comer & 50 & 12 & 25 & 6 & 25 & 6 \\
4- Tomar banho & 87,5 & 21 & 4 & 1 & 8,5 & 2 \\
5- Sair para dar uma volta & 29 & 7 & 50 & 12 & 21 & 5 \\
6- Subir degraus & 29 & 7 & 29 & 7 & 42 & 10 \\
8- Fazer esportes & 4 & 1 & 22 & 5 & 74 & 17 \\
9- Levantar 5 quilos & 27 & 3 & 36 & 4 & 37 & 4 \\
10- Correr & 0 & 0 & 25 & 6 & 75 & 18 \\
\hline
\end{tabular}

Tabela 2 - Descrição dos resultados obtidos em relação à dispnéia durante a realização de atividades de fala

\begin{tabular}{lcccccc}
\hline \multirow{2}{*}{ Atividades de fala } & \multicolumn{7}{c}{ Presença de Dispnéia } \\
\cline { 2 - 7 } & $\%$ & $\mathbf{N}$ & $\%$ & $\mathbf{N}$ & $\%$ & $\mathbf{N}$ \\
\cline { 2 - 7 } & 75 & 18 & 8 & 2 & 17 & 4 \\
\hline 1- Conversar com alguém & 79 & 19 & 21 & 5 & 0 & 0 \\
2- Falar ao telefone & 55 & 11 & 35 & 7 & & 2 \\
3- Cantar ou murmurar & 92 & 22 & 8 & 2 & 0 & 0 \\
4- Falar com TV ligada & 95 & 21 & 0 & 0 & 5 & 1 \\
5- Falar num restaurante & 57 & 13 & 26 & 6 & 17 & 4 \\
6- Falar para grupo de pessoas & 48 & 11 & 13 & 3 & 39 & 9 \\
7- Aumentar voz & 78 & 18 & 13 & 3 & 9 & 2 \\
8- Falar no carro & 67 & 16 & 25 & 5 & 8 & 2 \\
9- Falar em lugar ruidoso & 39 & 9 & & & & \\
10- Falar com autoridade & & &
\end{tabular}

Tabela 3 - Descrição dos resultados obtidos em relação à dispnéia durante a realização de atividades físicas associadas a atividades de fala

\begin{tabular}{lcccccc}
\hline \multirow{2}{*}{$\begin{array}{l}\text { Atividades Físicas } \\
\text { associadas a fala }\end{array}$} & \multicolumn{7}{c}{ Ausência } & \multicolumn{7}{c}{ Ocasionalmente } & \multicolumn{2}{c}{ Sempre presente } \\
\cline { 2 - 7 } & $\%$ & $\mathbf{N}$ & $\%$ & $\mathbf{N}$ & $\%$ & $\mathbf{N}$ \\
\cline { 2 - 7 } & 71 & 17 & 25 & 6 & 4 & 1 \\
\hline 1- Falar andando & 83 & 19 & 17 & 4 & 4 & 1 \\
2- Falar enquanto se veste & 83 & 20 & 13 & 3 & 4 & 1 \\
3- Falar andando no plano & 8 & 2 & 17 & 4 & 75 & 18 \\
4- Falar subindo ladeira & 25 & 6 & 21 & 5 & 54 & 13 \\
5- Falar subindo degraus & 70 & 15 & 15 & 3 & 15 & 3 \\
6- Falar/cantar tomando banho & 9 & 2 & 17 & 4 & 74 & 17 \\
7- Falar realizando esportes & 0 & 0 & 36 & 4 & 64 & 7 \\
8- Falar levantando 5 quilos & 4 & 1 & 25 & 6 & 71 & 17 \\
9- Falar enquanto corre & & & & & &
\end{tabular}




\section{DISCUSSÃO}

A respiração oral é uma síndrome decorrente de alterações primárias causadoras de obstrução nasal, como a asma e a rinite alérgica ou até mesmo de hábitos viciosos. Esta síndrome causa alterações em vários sistemas corporais, dentre eles o respiratório, sendo uma das queixas mais comuns do respirador oral ${ }^{10}$ a falta de ar ou insuficiência respiratória.

$\mathrm{Na}$ análise do questionário de dispnéia verificou-se na população estudada maior presença de queixa respiratória nas atividades físicas associadas a atividades de fala, seguida da realização de atividades físicas isoladamente e menor queixa de dispnéia durante as atividades de fala. Concordando com Lee ${ }^{17}$ que ao avaliar indivíduos com doenças respiratórias verificou a mesma ordem de ocorrência de dispnéia nas atividades pesquisadas. Em outro estudo que utilizou o mesmo questionário em indivíduos com Doença pulmonar obstrutiva crônica (DPOC) verificou-se maior queixa de dispnéia nas atividades físicas, seguidas das atividades físicas associadas à fala e menor queixa respiratória foi verificada nas atividades de fala ${ }^{16}$, demonstrando resultados semelhantes aos achados deste estudo.

No presente estudo verificou-se maior queixa respiratória na realização das atividades físicas associadas à fala, destacando-se falar subindo ladeira $(75 \%)$, realizando esportes $(74 \%)$, correndo $(71 \%)$, levantando peso (64\%) e subindo degraus (54\%). Dificuldade na realização das atividades de falar subindo ladeira e degraus em pacientes com doenças respiratória já foi observado em um estudo ${ }^{16}$.

As atividades de realizar esportes (74\%) e correr $(75 \%)$ foram as mais frequentemente relatadas com dispnéia freqüente dentre o grupo das atividades físicas. Tal dificuldade respiratória pode ser atribuída além da sonolência diurna, à modificação dos mecanismos de absorção dos gases, com diminuição do aproveitamento da oxigenação ${ }^{10}$, o que promove um maior esforço respiratório durante exercícios físicos, na tentativa de manter constantes as proporções gasosas no sangue.

Em relação às atividades de fala, apenas as atividades de falar com autoridade (39\%) e aumentar a voz (34\%), foram relatadas com dispnéia freqüente por alguns indivíduos. Sabe-se que o aumento da intensidade vocal depende do aumento de pressão aérea subglótica controlada pela adução glótica e pelo fluxo aéreo expiratório ${ }^{18}$. Pacientes com asma, rinite alérgica e respiração oral apresentam obstrução do fluxo aéreo e de fenômenos como hiper-responsividade e inflamação crônica das vias aéreas ${ }^{1}$. O indivíduo com asma, rinite alérgica e respiração tem dificuldade na inspiração e expiração, dificultando a realização adequada da respiração nessa atividade de fala ${ }^{16,19}$. As demais atividades de fala não foram relatadas com freqüência, provavelmente por serem atividades que não exigem esforço.

Vale ressaltar a literatura escassa sobre este assunto, o que prejudica a comparação destes achados com outros estudos realizados, daí a necessidade de estudos posteriores que possam avaliar e comparar os aspectos pesquisados nesta população.

\section{CONCLUSÃO}

Pode-se concluir em relação aos indivíduos pesquisados que as maiores queixas respiratórias foram encontradas nas atividades físicas associadas à fala, seguidas das atividades físicas isoladas. Já nas atividades comunicativas, apenas as atividades que exigiam maior intensidade vocal foram relatadas como dificultosas por alguns indivíduos.

Diante dos dados obtidos verifica-se que a respiração oral, além das alterações corporais, faciais, de aprendizagem e oclusão também acarreta prejuízos respiratórios aos indivíduos em situações que exigem maior capacidade respiratória. 


\begin{abstract}
Purpose: to evaluate the presence of respiratory dyspnea during physical activity, speech and its association. Method: the target population consisted of 24 patients with prior asthma medical diagnosis, allergic rhinitis and oral breathing, where we orally applied in each individual the Dyspnea Questionnaire translated by Cukier (1998), composed of 30 questions that allowed quantification of dyspnea activities in speech, physical and associates. Results: the analysis of the questionnaire showed higher respiratory complaints during the performance of physical activity associated with speech, followed by individual physical activities.. Conclusions: the major respiratory symptoms were found in physical activity associated with speech, followed by physical activity per se. Already in communicative activities, only activities that required more vocal intensity were reported to be hampered by some subjects.
\end{abstract}

KEYWORDS: Mouth Breathing; Asthma; Dyspnea

\section{REFERÊNCIAS}

1. Hetzel JL, Silva LCC. Tratamento atual da asma. In: Porto NS, Araújo E, Godoy DV, organizador. Doenças da vias aéreas: uma visão clínica integradora (viaerologia). Rio de Janeiro: Revinter; 2001.p.267-76.

2. Bousquet J. Aria Workshop Group. Recommendations. J Allergy Clin Immunol.2001; 108: 147-336.

3. ARIA. Manejo da rinite alérgica e seu impacto na asma: guia de bolso. ARIA no Brasil, 2002. 23p.

4. Heringer MRC, Reis M, Pereira LFS, Di Ninno CQMS. A influência da amamentação natural no desenvolvimento dos hábitos orais. Rev CEFAC. 2005; 7(3):307-10.

5. Ibiapina, CC; SARINHO, Sarinho ESC, Cruz Filho AAS, Camargos, PAM Moreira. Rinite, sinusite e asma:indissociáveis? J. Bras. Pneumol. 2006; 32(4): 357-66.

6. Barros JRC, Becker HMG, Pinto JA. Avaliação de atopia em crianças respiradoras bucais atendidas em centro de referência. J Pediatr. 2006; 82(6):458-64.

7. Craig TJ, Hanks CD, Fisher LH. How do topical nasal corticosteroids improve sleep and daytime somnolence in allergic rhinitis? J Allergy Clinical Immunol. 2005;116(6):1264-6.

8. Branco A, Ferrari GF, Weber SAT. Alterações orofaciais em doenças alérgicas de vias aéreas. Rev Paul Pediatr. 2007; 25(3): 266-70.

9. Queluz DP, Gimenes CMM. A síndrome do respirador bucal. Rev CROMG. 2000; 6(1):4-9.
10. Marchesan IQ. Avaliação e Terapia dos Problemas da Respiração. In: Marchesan IQ. Fundamentos em Fonoaudiologia - Aspectos Clínicos da Motricidade Oral. Rio de Janeiro: Guanabara Koogan, 1998. p. 23-36.

11. Breda D, Moreira HSB. Avaliação postural e da função respiratória em crianças com rinite alérgica, hipertrofia de adenóide e síndrome do respirador bucal. Fisioterapia Brasil. 2003; 4:247-52.

12. Campanha SMA, Freire LMS e Fontes MJF. O impacto da asma, da rinite alérgica e da respiração oral na qualidade de vida de crianças e adolescentes. Rev CEFAC.2008;10(4):513-9.

13. Menezes VA. Leal RB, Pessoa RS, Pontes RMES. Prevalência e fatores associados à respiração oral em escolares participantes do projeto santo AmaroRecife. Rev Bras Otorrinilaringol. 2006; 72(3):394-9. 14. Vera CFD, Conde GES, Wajnsztejn R, Nemr K. Transtornos de aprendizagem e presença de respiração oral em indivíduos com diagnóstico de transtornos de déficit de atenção/hiperatividade (TDAH). Rev CEFAC. 2006; 8(4):441-55.

15. Junqueira $P$, Parro FM, Toledo MR, Araújo RLT, Di Francesco R, Rizzo MC. Conduta fonoaudiológica para pacientes com diagnóstico de rinite alérgica: relato de caso. Rev CEFAC. 2005; 7(3):336-9.

16. Cukier S. Padrão respiratório durante a fala e parâmetros vocais de indivíduos com doença pulmonar obstrutiva crônica [monografia]. São Paulo: Centro de Estudos da Voz; 2003.

17. Lee L, Frisen M, lambert IR, Loudon RG. Evaluation of dyspnea during physical and speech activies in patients with pulmonary disease. Chest. 1998; 113(3):625-32. 
18. Pinho SMR. Avaliação e Tratamento da Voz. In: Pinho SMR. Fundamentos em Fonoaudiologia - Tratando os Distúrbios da Voz. Rio de Janeiro: Guanabara Koogan, 1998. p. 3-37.
19. Wertzener HF, Schreiber S, Amaro L. Análise da frequencia fundamental, jitter, shimmer e intensidade vocal em crianças com transtorno fonológico. Rev Brás Otorrinololaringol. 2005; 7(5):532-8.

Endereço para correspondência:

Silvia Márcia Andrade Campanha

Rua Penafiel, 84 ap 601

Belo Horizonte - MG

CEP: $30310-420$

E-mail: silviacampanha@ @otmail.com 